\title{
ON A PROBLEM OF KUROSCH AND JACOBSON
}

\section{IRVING KAPLANSKY}

1. Introduction. Let $A$ be an algebraic algebra over a field $K$, that is, an algebra over $K$ each of whose elements satisfies a polynomial equation with coefficients in $K$. In analogy to Burnside's problem for groups, Kurosch ${ }^{1}$ has raised the following question: if $A$ is finitely generated, does it necessarily have a finite basis? Jacobson ${ }^{2}$ studied the question for the case where the elements of $A$ are of bounded degree, and reduced it to the consideration of certain specific nil algebras defined as follows: $A(r, n)=F(r)-I(r, n)$, where $F(r)$ is the free algebra generated over $K$ by indeterminates $u_{1}, \cdots, u_{r}$, and $I(r, n)$ is the (two-sided) ideal generated by all $n$th powers in $F(r)$. In this note we shall prove the following theorem.

Theorem 1. If $K$ has at least $n$ elements, $A(r, n)$ has a finite basis.

Thus Kurosch's question for algebraic algebras of bounded degree receives an affirmative answer if $K$ is large enough, and in particular if it is infinite.

In $\S 3$, by a different method suggested by Kurosch's treatment of $n=3$, we prove that $A(r, 4)$ has a finite basis over $G F(3)$. In $\$ 4$ we discuss a special case of another question proposed by Jacobson: if the dimension $d(r, n)$ of $A(r, n)$ is finite, what is its precise value? We show that $d(2,3)$ may be equal to 16 or 17 , depending on $K$.

2. Proof of Theorem 1. Throughout this section we shall assume that the coefficient field $K$ has at least $n$ elements.

The algebra $F(r)$ consists of all (noncommutative) polynomials in the $u$ 's with coefficients in $K$ : that is, linear combinations of terms $u_{i} u_{j} u_{k} \ldots$ which we shall call monomials. The degree of a monomial is the number of $u$ 's it contains, and a polynomial is homogeneous if its monomials all have the same degree. We now prove the following lemma.

LEMMA 1. The ideal $I(r, n)$ has a basis of homogeneous polynomials.

Proof. Specifically, $I=I(r, n)$ has a basis consisting of all

Received by the editors February 13, 1946.

1 Ringtheoretische Probleme die mit dem Burnsideschen Problem über periodische Gruppen in Zusammenhang stehen, Bull. Acad. Sci. URSS. Ser. Math. vol. 5 (1941) pp. 233-240.

Structure theory for algebraic algebras of bounded degree, Ann. of Math. (2) vol. 46 (1945) pp. 695-707. 
$S\left(x^{i} y^{j} z^{k} \cdots\right)$ where $x, y, z, \cdots$ are monomials, $i+j+k+\cdots=n$, and $S$ denotes (as it will throughout the paper) the sum of products taken in all the $n ! / i ! j ! k ! \ldots$ possible orders. That these polynomials generate at least $I(r, n)$ follows from the fact that for any scalars $\alpha, \beta, \gamma, \cdots,(\alpha x+\beta y+\gamma z+\cdots)^{n}$ is a linear combination of such polynomials.

We show conversely that every $S\left(x^{i} y^{j} z^{k} \ldots\right)$ is in $I$. We have $T=(\alpha x+\beta y+\gamma z+\cdots)^{n} \in I$ for any scalars $\alpha, \beta, \gamma, \cdots$. Let $T_{i}=T_{i}(\alpha)$ be the sum of all terms in the expansion of $T$ which are of degree $i$ in $x$. Then $T=\sum_{i=0}^{n} \alpha^{i} T_{i}(1)$. Since $T_{0}$ and $T_{n}$ are in $I$, the sum from 1 to $n-1$ is likewise in $I$. Applying this with $n-1$ different nonzero values of $\alpha$, and multiplying by the minors of the resulting Vandermonde determinant, we see that $T_{i} \in I$. We now set $\alpha=1$ and let $T_{i j}=T_{i j}(\beta)$ be the subset of $T_{i}$ containing those terms of degree $j$ in $y$; then $T_{i}=\sum_{j=0}^{n-1} \beta^{i} T_{i j}(1)$. We may assume by induction on the number of terms $x, y, z, \cdots$ that $T_{i 0} \in I$. Taking $n-i$ different nonzero values for $\beta$, we obtain $T_{i j} \in I$. Continuing in this fashion, we prove that $T_{i j k} \ldots=S\left(x^{i} y^{i} z^{k} \ldots\right) \in I$.

Any element of $F(r)$ may evidently be written as a unique sum of homogeneous polynomials, which we may call its homogeneous parts. From Lemma 1 we have at once the following lemma.

LEMma 2. Any element of $F(r)$ is in $I(r, n)$ if and only if each of its homogeneous parts is in $I(r, n)$.

In order to prove Theorem 1 by induction, we prove a stronger result.

Lemma 3. Suppose that $B$ is an ideal in $A=A(r, n)$ generated by a finite number of ith powers of elements of $A$, and that $A-B$ is nilpotent. Then $A$ is nilpotent.

Proof. Lemma 3 is certainly true for $i=n$. We make a descending induction on $i$. Suppose $B$ is generated by $y_{1}{ }^{i}, \cdots, y_{m}{ }^{i}$ and that $(A-B)^{k}=0$. Let $M$ be the set of all monomials of degree less than $k(m+1)$. Let $C$ be the ideal generated by $y_{1}{ }^{i+1}, \cdots, y_{m}{ }^{i+1}$, and by all $x^{i+1},(y+\alpha x)^{i+1}$, where $y$ ranges over $y_{i}, x$ ranges over $M$, and $\alpha$ runs through $i$ different nonzero scalars. Use of the Vandermonde determinant shows that

$$
S\left(y^{i} x\right)=y^{i} x+y^{i-1} x y+\cdots+x y^{i}
$$

is in $C$. Right-multiplying by $y^{i}$, we have $y^{i} x y^{i} \in C$.

We shall now show that $A-C$ is nilpotent, whence by induction $A$ is. Let $t$ be any monomial of degree $k(m+1)$. We may write 
$t=z_{1} z_{2} \cdots z_{m+1}$, with each $z$ a monomial of degree $k$. Since $(A-B)^{k}=0$ each $z$ is in $B$ and can be written as a sum of terms $a y^{i} b$; moreover by Lemma 2 we may assume that each of these terms $a y^{i} b$ has degree $k$. Now in a product of $m+1$ terms, each of the form $a y^{i} b$, at least one $y$ must get repeated; hence such a product contains $y^{i} x y^{i}$ with $x$ a monomial of degree less than $k(m+1)$. As shown above, $y^{i} x y^{i} \in C$, whence $t \in C$, and $A-C$ is nilpotent of index at most $k(m+1)$.

Theorem 1 is an immediate consequence of Lemma 3. We take the case $i=1, B=\left(u_{1}, \cdots, u_{r}\right)=A$. The hypothesis that $A-B$ is nilpotent is fulfilled, and we conclude that $A$ is nilpotent and has a finite basis.

3. The case $n=4$. Kurosch gave a direct combinatorial proof that $A(r, 3)$ has a finite basis, which, unlike the above proof, is also valid over $G F(2)$. We shall show that his argument can be extended so as to push the case $n=4$ nearer completion.

THEOREM 2. $A(r, 4)$ has a finite basis over $G F(3)$.

Proof. Define

$$
\begin{aligned}
f(x, y, z)= & (x+y+z)^{4}-(x+y)^{4}-(x+z)^{4} \\
& -(y+z)^{4}+x^{4}+y^{4}+z^{4}
\end{aligned}
$$

Then

$$
x^{3} y x^{3}=x^{2}\left[f\left(x, y, x^{2}\right)+f\left(-x, y, x^{2}\right)-x^{2} y x^{2}-x^{3} y x\right]=0 .
$$

Hence the (two-sided) ideal generated by $x^{3}$ is nilpotent. More generally, any ideal $B$ generated by a finite number of cubes is nilpotent. To show that $A=A(r, 4)$ is nilpotent it therefore suffices to prove $A-B$ nilpotent, that is, we may take any finite number of cubes to be zero without loss of generality.

We may suppose by induction that the subalgebra $C$ generated by $u_{1}, \cdots, u_{r-1}$ has a finite basis $\left\{t_{i}\right\}$, and let us write $u$ for $u_{r}$. Set $u^{3}, t_{i}{ }^{3}$, and $\left(u \pm t_{i}\right)^{3}$ all equal to zero. Then $u^{2} t_{i}+u t_{i} u+t_{i} u^{2}=0$, whence for any $x \in C$

$$
u^{2} x+u x u+x u^{2}=0,
$$

and right-multiplying by $u^{2}$ we have

$$
u^{2} x u^{2}=0 \text {. }
$$

Using (1) to replace $u x u$ by $-u^{2} x-x u^{2}$ and then using (2), we see that any monomial containing four $u$ 's vanishes. This proves the theorem. 
4. The order of $A(2,3)$. In the case $n=2$, we have $(x+y)^{2}=0$ and hence $x y=-y x$ for any $x, y$. In the case of characteristic 2 , $A(r, 2)$ is commutative, and its order $d(r, 2)$ is $2^{r}-1$ as observed by Jacobson. ${ }^{3}$ For characteristic not equal to 2 , we have $(x y) z=-z(x y)$ but also $x(y z)=-x(z y)=z(x y)$, so that $x y z=0$. It follows that the elements $u_{i}$ and $u_{i} u_{j}(i<j)$ constitute a basis, that is, $d(r, 2)=r+{ }_{r} C_{2}$ $={ }_{r+1} C_{2}$.

We shall now evaluate $d(r, n)$ in the next simplest case: $r=2, n=3$. The result seems to indicate that a complete evaluation of $d(r, n)$ will involve formidable combinatorial difficulties.

THEOREM 3. If the coefficient field is GF(2) or if it has characteristic 3, then $d(2,3)=17$; otherwise $d(2,3)=16$.

Proof. From $(x+y)^{3}=0$ we obtain

$$
S\left(x^{2} y\right)+S\left(x y^{2}\right)=0
$$

and from

$$
\begin{aligned}
(x+y+z)^{3}= & (x+y)^{3}+(x+z)^{3}+(y+z)^{3} \\
& +S(x y z)-x^{3}-y^{3}-z^{3}
\end{aligned}
$$

we have

$$
S(x y z)=0 .
$$

Take $x=u, y=v, z=u^{2}$ in (5):

$$
u v u^{2}+u^{2} v u=0 .
$$

Together with

$$
S\left(u^{2} v\right)+S\left(u v^{2}\right)=0
$$

this gives

(7) $S\left(u^{2} v\right)$ and $S\left(u v^{2}\right)$ are annihilated by $u$ and $v$ on both left and right. Next, by taking $x=u, y=v, z=u v$ in (5) and using (7), we have

$$
v u v u=u^{2} v^{2} \text {, }
$$

which implies

$$
u v u v u=v u v u v=0 .
$$

From (7), (8), and (9) it is easy to see that all monomials of degree $\geqq 7$ vanish.

\footnotetext{
${ }^{3}$ Loc. cit. p. 707.
} 
Finally take $x=u, y=v, z=u v u v$ in (5) and make repeated use of (7) and (9). We find

$$
3 u^{2} v u v^{2}=0 .
$$

We now assert that for $K=G F(2),(6)-(11)$ is an exhaustive set of relations on $u, v$; for $K \neq G F(2),(6)$ and (7) may be strengthened to

$$
S\left(u^{2} v\right)=S\left(u v^{2}\right)=0,
$$

and (8)-(12) is an exhaustive set of relations. To prove this it suffices to assume the relations in question, and show that they ensure the evanescence of every cube. For the latter it suffices to prove that the cube of any monomial vanishes and that (3) and (5) hold for any monomials $x, y, z$. By (10), there are only a finite number of verifications to make; we omit the details of the computation.

We can now make a list of basis elements. It is convenient to note, by systematic use of ( 7$)$, that candidates for basis elements of degree not less than 4 need contain $u^{2}\left(v^{2}\right)$ only at the beginning (end).

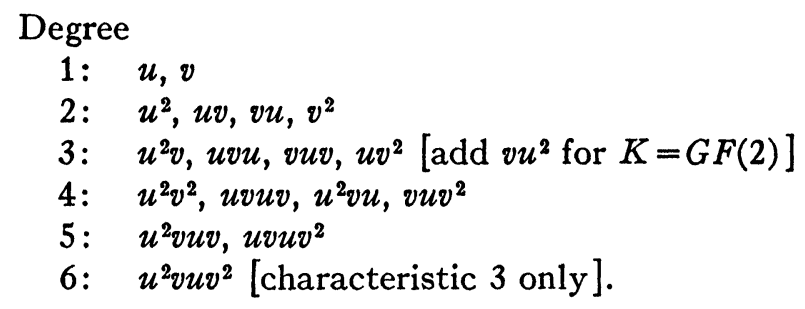

Added in proof: Professor Jacobson has pointed out to me that (by $\$ 9$ of his paper) an affirmative answer to Burnside's problem for fourth powers would imply that $A(r, 4)$ has a finite basis over $G F(2)$, and thus complete the case $n=4$. Moreover the known affirmative answer for the case of two generators shows that $d(2,4)$ over $G F(2)$ is finite, and has the explicit (though probably much too large) upper bound of 1024 .

University of Chicago 